\title{
Traduire
}

Une autre perspective sur r tr traduction

Revue française de la traduction

$241 \mid 2019$

La formation à l'honneur

\section{Transmettre pour mieux transcrire, et inversement}

\section{Dominic Michelin}

\section{OpenEdition \\ Journals}

Édition électronique

URL : http://journals.openedition.org/traduire/1866

DOI : 10.4000/traduire.1866

ISSN : 2272-9992

\section{Éditeur}

Société française des traducteurs

\section{Édition imprimée}

Date de publication : 16 décembre 2019

Pagination : 81-90

ISSN : 0395-773X

\section{Référence électronique}

Dominic Michelin, «Transmettre pour mieux transcrire, et inversement», Traduire [En ligne], 241 | 2019, mis en ligne le 16 décembre 2019, consulté le 06 février 2020. URL : http://journals.openedition.org/ traduire/1866 ; DOI : 10.4000/traduire.1866 


\section{Transmettre pour mieux transcrire, et inversement}

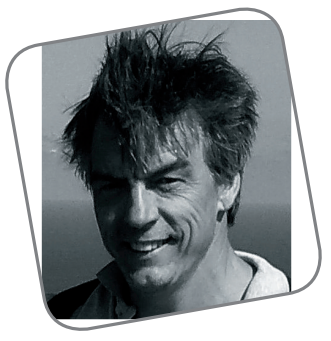

\section{Dominic Michelin}

\section{Préambule}

Comment devient-on traducteur? Avant de s'interroger sur la formation et l'itinéraire qui mènent aux métiers de la traduction, on peut se demander qui et ce qu'est un traducteur ou une traductrice. Il existe à l'évidence une infinité de profils du métier. Le fait que la profession n'est - toujours - pas réglementée implique qu'aucune formation spécifique ne s'impose. Pourtant, certaines parmi les plus éminentes et les plus anciennes de haut niveau (ESIT et ISIT, 1957; Inalco, exécole spéciale des langues orientales, 1795) se sont fait une place naturellement. Celles que proposent les masters affiliés EMT, souvent articulés sur l'excellent mode de l'alternance, sont nées pour la plupart au tournant du dernier siècle, et les formations spécifiques encore plus récentes (CI3M, 2009) coexistent depuis lors. La tendance croissante de la formation diplômante du métier sur le vaste marché de la traduction prévaut, comme le confirment, d'une part, le nombre de formations diplômantes offertes et, d'autre part, le profil des traducteurs arrivés sur le marché ces dix dernières années, ce qui est mon cas. 
S'il apparaît logique de se former a minima pour exercer, puis en continu tout au long de sa carrière une fois l'activité devenue viable, il m'a paru naturel à peine installé de devenir aussi transmetteur d'expérience, passeur de connaissances pour les générations proches ou les vocations en devenir. Ma formation initiale étant encore récente, la transmission devenait d'autant plus génératrice d'intérêt et d'enthousiasme. Si indubitablement "enseigner, c'est apprendre», être en situation d'enseignant, de formateur ou de transmetteur est aussi une position simultanément et éminemment délicate et valorisante: tout à la fois un défi, un plaisir, une remise en question, une expérience, une mise à nu, mais aussi une complicité, une association, une passerelle bâtie et tendue vers le futur pour soi et pour les praticiens du métier en devenir. Enfin, lorsqu'on transmet, l'idée est aussi de faire connaître, d'incarner un métier méconnu ou trop souvent réduit à ses clichés.

\section{Envolée}

S'il n'existe aucune obligation légale de formation, le traducteur indépendant en fait le choix afin de se faire une place, voire sa place, et ce indépendamment du fait qu'il exerce sous forme de micro-entreprise, de coopérative, de profession libérale ou d'artiste-auteur. L'espace que constitue le marché de la traduction dans sa grande diversité, dans tout son aspect concurrentiel, autant que solidaire, généreux et complémentaire demeure, en effet, très vaste.

En ce qui me concerne, j'ai la sensation d'avoir toujours eu envie d'être traducteur, ou en tout cas de voyager et d'exister par les langues et partant, par les cultures. La réalisation de mon projet a néanmoins dû attendre le moment idoine, à savoir un contexte économique et personnel propice. II s'est présenté il y a une dizaine d'années, après avoir travaillé quinze ans pour un grand groupe du secteur aéronautique, et avoir voyagé et vécu dans différents pays. J'ai abordé ce virage quasi existentiel non sans avoir consciencieusement choisi les moyens et le cadre de ma (re)conversion, à savoir une formation professionnelle appropriée et un diplôme d'études supérieures obtenu en alternance et suffisamment spécialisé pour acquérir les techniques a priori ad hoc et me familiariser avec les technologies les plus récentes. 
Mon intention première était d'être en contact avec des intervenants professionnels en activité, clé de réussite d'un lancement opérationnel. L'immense diversité du marché de la traduction est ce qui en fait tout à la fois le charme et l'enjeu. Il faut être en permanence en veille, prendre des décisions mesurées, rapides et efficaces, être à la fois solitaire de profession et solidaire du métier, être prêt à se diversifier pour varier les plaisirs et élargir ses compétences, se risquer sur de nouveaux sentiers ou avec de nouveaux équipiers et se lancer à l'assaut de nouveaux domaines de spécialité, goûter ou re-goûter à l'interprétation.

En tenant compte de la diversité de disciplines et de fonctions que peut déployer la profession de traducteurinterprète, il m'est apparu essentiel de me positionner en passeur-vecteur de connaissances et d'informations utiles et pratiques. Différentes activités autant sujettes à défi que valorisantes y contribuent: formation, présentation, délégation, promotion et participation.

Je ne me suis lancé qu'une fois armé de connaissances théoriques et d'outils pratiques, et nourri des expériences de pairs en activité et fort de leur appui complétant mon propre vécu international et interculturel. Et mon pressentiment s'est vite confirmé, à savoir que le métier de traducteur-interprète indépendant fait appel à l'ensemble de nos expériences et impose de les mettre en pratique: s'adapter à la situation ainsi qu'à l'interlocuteur, éprouver les différents aspects de l'entreprise individuelle ainsi que son relationnel avec l'ensemble des protagonistes. C'est pourquoi il est important de savoir promouvoir et valoriser le rôle absolument essentiel de notre activité, en communiquant et en transmettant au mieux notre savoir, qu'il soit théorique ou empirique.

De cette association d'expériences, j'ai tiré la conviction qu'il est, sinon impératif, en tout cas éminemment positif de les associer au travail de traducteur-transmetteur.

\section{Transport de sens}

Avant d'aller plus loin dans cette logistique de transmission linguistique et culturelle, rappelons ici la valeur étymologique même du mot «traduction»: du latin traductio, "traversée, action de faire passer d'un point à un autre». 
En effet, loin de se confiner à un rôle déjà crucial de messager du sens des mots et des cultures, le traducteur devient l'ambassadeur d'un concept innovant, d'un néologisme, d'un terme oublié ou inusité, d'une spécificité culturelle, voire d'un trait de caractère, souvent issu de l'usage ou participant d'une certaine logique linguistique. C'est le cas de mots tels que "états-unien», "riverainisme», "zénitude», ou encore «trophologie» que le traducteur intègre à son œuvre du moment pour la rendre aussi temporelle que possible. Parallèlement, nombre de mots étrangers cherchent encore leur traduction en français, parmi lesquels je vous propose une sélection pour éprouver votre créativité (voir encadré en fin d'article).

Aussi, en situation de formateur ou de transmetteur, je m'efforce de sensibiliser mon audience sur les aspects suivants par le biais de situations, d'exemples ou d'anecdotes:

- Combien se recoupent et comment peuvent s'utiliser nos expériences en terminologie, sur les registres de langue, les relations humaines, l'approche d'un nouvel outil, la façon de se présenter, de promouvoir ou d'évoquer son activité en quelques minutes lors d'une rencontre ou d'une discussion impromptue par un argumentaire éclair (le fameux elevator pitch) ou via sa plaquette de référence.

- Quelles peuvent être les leçons à tirer de mésaventures liées au sens, à la situation ou au relationnel? Dans un contexte de formation ou de transmission, comment intégrer du ludisme, technique d'apprentissage toujours efficace, par le biais de quiz, ou de réflexions sur des termes a priori intraduisibles pour enseigner, transmettre le message et les techniques et faire passer l'aspect émotionnel d'un texte qui doit d'abord se «sentir»dans la langue source avant d'être transposé.

- "Enseigner, c'est apprendre.»Cette démarche se révèle éminemment profitable pour présenter une difficulté de traduction; énoncer cette dernière apporte souvent un début de solution. D'où l'importance de l'échange avec ses pairs, avec les experts ou les locuteurs de la langue source.

- Transmettre son expérience et ses connaissances sous forme d'anecdotes et d'expériences, comme je l'ai fait 
au départ en proposant un tutorat à des étudiants, en présentant mon activité lors de forums en université ou dans le cadre de cercles d'entrepreneurs indépendants, puis en travaillant comme directeur de mémoires en traduction.

- Sortir de sa zone de confort intellectuelle, physique ou sociale, en cherchant et en trouvant les mises en situation génératrices d'enjeux personnels et professionnels, que ce soit en mission occasionnelle d'interprétation ou dans le cadre d'évènements spécifiques comme les salons spécialisés ou de manifestations dédiées de près ou de loin au métier.

\section{Croisière et croisade}

«Tous les jours, des milliards de messages sont diffusés sur tous supports et en plusieurs langues, véhiculant des idées nouvelles, des dogmes et des croyances, des principes et des jugements de valeurs, des codes et des normes. Ils fabriquent une culture universelle qui s'élabore par la traduction, dans le contact permanent des langues et des cultures ${ }^{1}$.»

D'aucuns diraient que l'activité essentielle du traducteur consiste à traduire, donc à transposer, à écrire et à réécrire en tapant sur son clavier un certain nombre de pages pour produire un texte clair, fluide, explicite et de préférence agréable pour les futurs lecteurs qui le découvriront en contexte et oublieront que ce texte fut autrefois autre, presque étranger. Traduire finit par devenir une quasi-habitude, un mode de vie qu'heureusement chaque nouveau texte à retranscrire, à réécrire ou à interpréter vient bousculer, renouveler par son style, son contexte, sa technicité et la perception finale qu'on souhaite lui donner (exemples ci-après). La traduction devient logiquement et spontanément une écriture, pendant et après la délicate phase de la relecture à ne jamais négliger. II s'agit de rendre la traduction invisible en rendant le texte éminemment visible et naturellement lisible. C'est là où la phase de relecture (double, triple, voire multiple), que je ne cesse de rappeler fondamentale, prend toute sa valeur 
en accouchant finalement d'un texte fluide détaché de la traduction, vivant et indépendant.

Incontestablement, lâcher le texte source est difficile. On largue les amarres pour se retrouver dans une mer d'une perception différente, d'une couleur ou plutôt d'une tonalité autre dont on va apprendre à connaître enfin toutes les nuances. Certaines sont connues, ou semblent l'être, et d'autres sont des découvertes, voire des redécouvertes interpellant son propre vécu, qui souvent influe alors sur le choix des mots. Le plaisir de l'inspiration naît à cette étape, jusqu'à la remise plaisamment opportune de la clé des champs sémantiques.

"Malheur aux faiseurs de traductions littérales, qui en traduisant chaque parole énervent le sens! C'est bien là qu'on peut dire que la lettre tue et le sens vivifie.» (Voltaire, Lettres philosophiques, 1734)

\section{Du choix et du sens des mots pour transporter à destination}

Dans les extrêmes de médiatisation, d'interconnexion et de globalisation actuels, la vitesse et la synthèse de la communication telle qu'elle est consommée rendent parfois imperceptible le sens premier des mots utilisés; c'est là qu'intervient la qualité d'une traduction aboutie et invisible. Pour preuve, l'influence, très souvent inconsciente pour le lecteur, que peut avoir le choix de certains mots, formules et expressions idiomatiques, choix pour lequel le traducteur conserve une aptitude et une latitude certaines. Combien compte le choix du style et du registre de langue pour rendre l'ambiance, l'atmosphère, le vécu, le ressenti, avant même de penser aux mots et aux termes adéquats. Entre fusion et tension interlinguistiques, la traduction porte une sensibilité et une subtilité qui lui confèrent d'étranges pouvoirs.

La qualité d'un traducteur doit transparaître dans sa façon de répondre, de s'exprimer, lors d'échanges allant du courrier électronique à son message de répondeur en passant par son élocution, sa présentation et son regard sur les autres et sur le monde. Le traducteur contribue en effet grandement, par le choix judicieux de mots et d'expressions comprises du plus grand nombre, à la qualité d'une expression 
écrite à laquelle les lecteurs, avisés ou non, sont sensibles. La mise en circulation d'un néologisme est souvent le fait et la responsabilité des traducteurs, ouvriers et orfèvres des mots, techniciens de la formulation et de l'ordonnancement et, finalement, ingénieux ingénieurs sociologiques discrets et efficaces.

Un exemple? Ainsi progresse l'usage naturel du terme - cité plus haut - "états-unien»... Car il est vraiment temps de mettre fin à un impérialisme néologique, vieux de plus de deux siècles, qui voit un pays s'approprier à lui seul un qualificatif pourtant étymologiquement bicontinental: américain.

L'impact de la lecture d'un terme à la place d'un autre, en $y$ incluant le son, et par conséquent, celui de toute la phrase dans lequel il résonne demeure stupéfiant. Un autre exemple? J'ai ainsi incidemment (vous l'entendez, là, l'écho?) modifié le titre d'un chapitre dans une bible du parfait management: "Choisir la bonne direction» est devenu "Tenir le cap», ce qui change sensiblement la perception de l'action qui de passive ou d'élective se fait active et qui plus est, sélective. Et cette perception est d'autant plus forte quand on vit/lit la suite: "Tenir le cap: loin de l'individualisme et vers la générosité et la recherche de l'intérêt commun».

Autre exemple dans un texte pour un guide de voyage: l'utilisation dans un texte d'environ une page des trois mots «escapade», "escalade» et "escarpement»pour la résonance du texte et la «complicité» avec le lecteur présumé aventurier.

Enfin, un tout dernier exemple qui illustre à nouveau la tonalité d'une allitération (en italique ci-dessous) tirée d'un texte évoquant la spécificité gastronomico-culturelle d'une recette britannique, utilisé lors d'un «traduel»:

Though the very idea of it strikes fear into the healthily functioning heart of every lily-livered, low-carb fitness influencer, the Cornish pasty is, quite simply, happiness in your hand. It's even shaped like a smile.

Si le concept même peut faire défaillir n'importe quel fringant thuriféraire de la trophologie vouant aux gémonies les glucides, la Cornish pasty n'en constitue pas moins un pur bonheur en soi, en témoigne son si séduisant galbe. 


\section{En quête de destination finale}

À l'instar d'un périple réussi au cœur de jungles idiomatiques, une traduction aboutie, loin de se limiter à la transposition ou à la transcription textuelle, nécessite un travail de préparation, d'appropriation pour appréhender, puis délivrer le sens profond d'un texte, pour capter l'équilibre subtil entre le fil conducteur d'une œuvre écrite et ses constructions, jeux de mots et autres clins d'œil culturels ou techniques.

De fait, rendre intelligible un texte de traduction issu d'un domaine spécialisé n'est pas uniquement affaire de spécialiste, mais bien une combinaison harmonieuse: appréhension du domaine, connaissance linguistique spécifique, bon sens et instinct, sélection judicieuse des bagages nécessaires et une faculté d'adaptation pour parvenir à la retranscription. Notre mission reste de mener le juste sens à bon port, de le rendre naturellement intelligible et fluide aux yeux du lecteur.

Pour conclure, le fait de m'être positionné rapidement en situation sensible de passeur d'expériences, de connaissances, d'anecdotes, d'épisodes, d'aventures, d'incidents, de scénarios et de situations propres à l'activité de traduction m'a permis d'acquérir et de partager un certain nombre d'idées inspirantes, voire de certitudes, qu'elles soient terminologiques, sociologiques, pratiques ou conceptuelles, d'autant qu'elles se sont nourries de ces échanges constructifs et surtout révélateurs d'une certaine place du métier de traducteur, assurément bien moins solitaire qu'il n'y paraît pour qui est prêt à s'en départir par le biais, ou plutôt le prisme de la transmission. En cela, la façon d'écrire, la forme de l'écriture, la résonance du style, associées directement ou indirectement constituent un canevas significatif du mode de perception et de rendu d'un texte et représentent toutes des éléments constitutifs essentiels à une traduction, cet exercice littéraire, téméraire et parfois arbitraire de ressentir le monde ou en tout cas de l'interpréter et d'en rendre au mieux cette perception, qui offre l'accès à des œuvres écrites dans d'autres langues, en dépit de sa transparence pour la plupart des lecteurs. Et c'est en transmettant ce savoir et cette expérience que le formidable métier de traducteur, invisible et incontournable, gagnera progressivement la reconnaissance et la notoriété qui lui sont dues. 


\section{INTRADUISIBLE, VOUS AVEZ DIT INTRADUISIBLE?}

iktsuarpok (inuit) Sensation mêlée d'impatience ressentie et exprimée physiquement par quelqu'un attendant quelqu'un sur le point d'arriver et scrutant l'extérieur ou sortant régulièrement pour vérifier l'arrivée de la personne attendue.

komorebi (japonais) Lumière du soleil filtrant à travers les feuilles des arbres, et par extension, tout rai de lumière filtrant à travers la matière.

pochemuchka (russe) Du russe почемý (počemú), «pourquoi». Enfant qui pose systématiquement des questions. Par extension, toute personne trop curieuse posant trop de questions et s'exposant à des représailles.

sobremesa (espagnol) Le temps qu'on passe à table, après un repas, à discuter entre convives.

sisu (finnois) Trait de caractère résumant le cœur de l'âme finlandaise, l'acharnement patient qui permet aux Finlandais d'affronter toutes les situations et de mener à bien leurs objectifs.

cafuné (portugais brésilien) Désigne ce geste tendre de quelqu'un qui passe sa main et fait glisser ses doigts dans les cheveux de quelqu'un d'autre en signe d'affection et/ou de réconfort.

hygge (danois/norvégien) État d'esprit positif, sensation de bien-être, de sécurité et de paix que l'on ressent dans un endroit particulier, dans une atmosphère intime, en compagnie de quelqu'un. Par extension, art de vivre, attitude positive malgré les circonstances.

desenrascanço (portugais) Littéralement «démêlage, dégagement»: faculté ou possibilité de se sortir d'une situation compliquée, d'improviser une solution sur le coup.

litost (tchèque) Racine étymologique associant pitié et cruauté: état de tourment intérieur, de tristesse provoqué par un sentiment de frustration et d'humiliation ressenti dans certaines situations pour soi-même pouvant se transformer en jalousie, voire en haine à l'égard des autres perçus comme étant la source de cet état et de ses conséquences.

saudade (portugais) Sentiment éprouvé pour quelqu'un ou quelque chose que vous aimez, mais qui est malheureusement perdu pour toujours.

mamihlapinatapai (yagan, langage indigène de la Terre de Feu) Désigne un regard plein de signification et de désir, partagé entre deux personnes dont chacune espère que l'autre va prendre l'initiative de déclencher ce que les deux désirent, mais qu'aucune ne se résout à faire. 
Dominic Michelin est traducteur indépendant depuis 2011. De formation littéraire et linguistique initiale, il a enseigné le français langue étrangère avant de se spécialiser en commerce international, puis en communication internationale et traduction spécialisée. II a notamment été responsable communicationmarketing pour une grande entreprise aéronautique. Dominic est diplômé de l'université de Dijon-Bourgogne (master en stratégies de communication internationale, 2001), de l'université Paris 7 et de I'ISIT (master en industrie des langues et traduction spécialisée, 2010).

SOURCES CITÉES

https://fr.babbel.com/fr/magazine/intraduisible-1

https://www.lemonde.fr/big-browser/article/2016/05/13/mamihlapinatapaishibumi-ces-mots-intraduisibles-qui-decrivent-nos-sentiments_ 4919406_4832693.html

https://europeisnotdead.com/fr/mots-intraduisibles-europeens/

www.topito.com/top-mots-intraduisibles

https://www.bedetheque.com/serie-18012-BD-Litost.html 